\title{
Coupled Brownian Motors
}

\author{
C. Van den Broeck ${ }^{1}$, P. Reimann ${ }^{2}$, R. Kawai ${ }^{3}$, and P. Hänggi ${ }^{2}$ \\ 1 Limburgs Universitair Centrum, B-3590 Diepenbeek, Belgium \\ 2 Theoretische Physik I, Universität Augsburg, D-86135 Augsburg, Germany \\ 3 University of Alabama at Birmingham, Birmingham, AL 35294, USA
}

\begin{abstract}
A unifying view on several astonishing effects induced by state dependent, uncorrelated fluctuations (multiplicative white noise) is presented. The main emphasis of our introductory discussion is put on the basic physical mechanisms at work in a white noise driven "pulsating ratchet", noise-induced non-equilibrium phase transitions, and their synthesis in the form of coupled Brownian motors.
\end{abstract}

\section{Introduction}

Can one get work out of fluctuations? Of course, one can! That is obvious in the case of (almost) periodic fluctuations: Think of all the rectifiers, mechanical, electrical and others, that allow a device to move only in one direction and hence build up energy. One of the long-known examples in the case of random fluctuations (unbiased on average but still correlated in time) is the wind-mill. The self-winding wristwatch is a more modern version that works especially well with native Italian speakers. But even unbiased fluctuations without any appreciable correlation in time (white noise) can be exploited to do useful work, as we will show below.

The above examples refer to macroscopic fluctuations. For microscopic fluctuations, the situation is more subtle. Indeed, we know from the second law of thermodynamics that it is impossible to get work in a repetitive cyclic manner from a single heat bath at equilibrium. The fact that rectifiers will not work in this situation is nicely illustrated in the ratchet-and-pawl example discussed by Feynman, Leighton, and Sands 1963 (see also the critical discussion by Parrondo and Espanol 1996 and a much earlier account of very similar ideas, that may have inspired Feynman, by Smoluchowski 1912). But nothing forbids the appearance of Maxwell demons, or Brownian motors (Vale and Oosawa 1990, Ajdari and Prost 1992, Magnasco 1993, Astumian and Bier 1994, Doering, Horsthemke, and Riordan 1994, Bartussek, Hänggi, and Kissner 1994), to use a more benign nomenclature, in the case of nonequilibrium fluctuations (for reviews see also Hänggi and Bartussek 1996, Astumian 1997, Jülicher, Ajdari, and Prost 1997). Indeed, Feynman's ratchet-and-pawl, as well as many other kinds of "heat-engines", if properly connected with two heat baths at different temperatures, do produce work, and this nota bene out of practically uncorrelated microscopic fluctuations. We further mention that biological systems are typically operating far from equilibrium and the 
small scale of the intracellular processes implies that fluctuations may play a significant role. In fact, Brownian motor models have been proposed as a possible mechanism for intracellular transport processes by Vale and Oosawa 1990, Ajdari and Prost 1992, Magnasco 1993, Astumian and Bier 1994, Doering, Horsthemke, and Riordan 1994, Astumian 1997, Jülicher, Ajdari, and Prost 1997. It is remarkable and little known that directed transport induced by unbiased driving forces in periodic structures with broken spatial symmetry (ratchets) has also been investigated theoretically and experimentally in the context of photovoltaic and photorefractive effects in noncentrosymmetric ferroelectrics such as $\mathrm{BaTi0}_{3}$ already for several decades, see the reviews by Belinicher and Sturman 1980 and by Sturman and Fridkin 1992. Likewise, directed current (or, equivalently, a finite voltage under open circuit conditions) emerges in symmetric periodic structures when driven by unbiased harmonic mixing signals, $E(t)=E_{1} \cos (\Omega t)+E_{2} \cos (2 \Omega t)$, due to the nonlinear static response of nonvanishing odd higher moments of order $n \geq 3$, see Breymayer, Risken, Vollmer, and Wonneberger 1982, Wonneberger and Breymayr 1984. These voltages have been observed experimentally as early as in 1978 by Seeger and Maurer.

To introduce the specific type of Brownian motor that will interest us, namely the "pulsating ratchet", we start with an amusing discrete version that shows how Maxwell demons can make money (J.M.R. Parrondo, private communication; related models, but formulated in a physical context, have been studied by Sokolov and Blumen 1997 and by Schimansky-Geier, Kschischo, and Fricke 1997). We consider two fair games, cf. Fig.1. The first one is coin tossing. When head comes up, the demon's capital increases by one, and yours decreases by one, and vice versa for tail. The other game is slightly more complicated: the demon wins with a probability of $2 / 3$ when his capital is equal to 1 or 2 , modulo 3 , and only with a probability of $1 / 5$ in the other cases, when his capital is 3 , modulo 3 . The most convincing way of showing to the layman that this game is fair is by trying it out: on average gains and losses cancel exactly. A physicists way to check it is by remembering the historic paper by Onsager 1931 on detailed balance, showing that micro-reversibility implies for a circular reaction at equilibrium, $c$. Fig.1, the result that the product of forward and backward rates should be equal: $k_{1}^{+} k_{2}^{+} k_{3}^{+}=k_{1}^{-} k_{2}^{-} k_{3}^{-}$. For the (discrete-time) Markov process corresponding to our game, this condition of detailed balance is indeed fulfilled: $\frac{2}{3} \frac{2}{3} \frac{1}{5}=\frac{1}{3} \frac{1}{3} \frac{4}{5}$. One can easily verify that any transition between adjacent states is then equally likely to occur in both directions.

Let us now play these games. After some time, one gets bored with game 1 and switches to game 2. As one keeps on switching randomly between the two games, it will become clear that the demon is making money and you are losing it. Switching at random between fair games is no longer fair! In fact, even after modifying the single games slightly in your favor, the demon will continue to win! To understand intuitively what is happening 


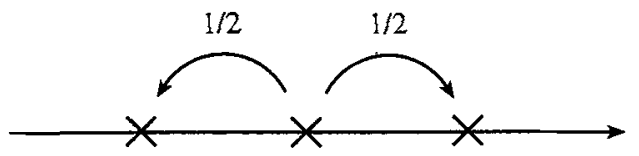

game 1

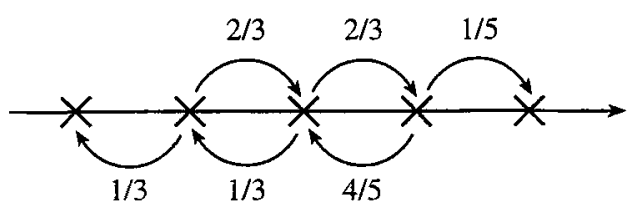

game 2

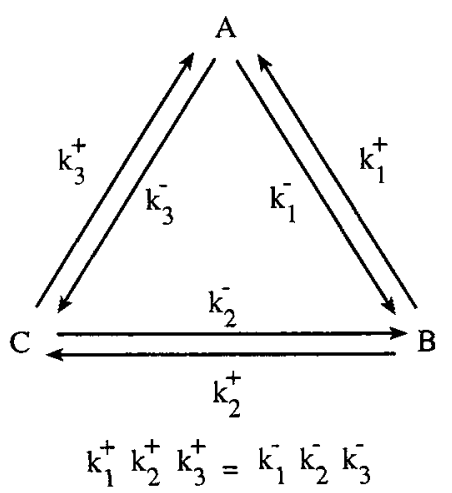

Fig. 1. Schematic representation of two fair games, and the corresponding circular chemical reaction for the second game in the spirit of Onsager. As detailed in the main text, random switching between the two fair games leads to a game that is no longer fair since the property of detailed balance is lost.

we turn to a continuum analogue of the two games, see Fig.2. The first game is just an unbiased random walk, which we replace by plain diffusion (free Brownian motion). The second one can be represented by Brownian motion in a sawtooth potential. The small slope, favoring motion to the right, extends over a longer interval than the large slope, which favors more strongly the motion to the left. The condition for detailed balance, implying the absence of a systematic motion to the right or left, requires here that the overall potential is not tilted: the total (slow) decrease of the potential 
over the longer interval has to exactly cancel the total (fast) increase of the potential over the smaller interval. The fact that this is the right condition is maybe not immediately obvious (it is actually just the point of Feynman, Leighton, and Sands 1963). It helps to remember that statistical mechanics predicts $Z^{-1} \mathrm{e}^{-\beta V_{0}(x)}$ as steady state distribution for Brownian particles at temperature $T$ (with $\beta=1 / k_{B} T$ ) in a potential $V_{0}(x)$. Clearly, in the case of a tilted potential, particles would constantly be moving in the direction of the decreasing potential.

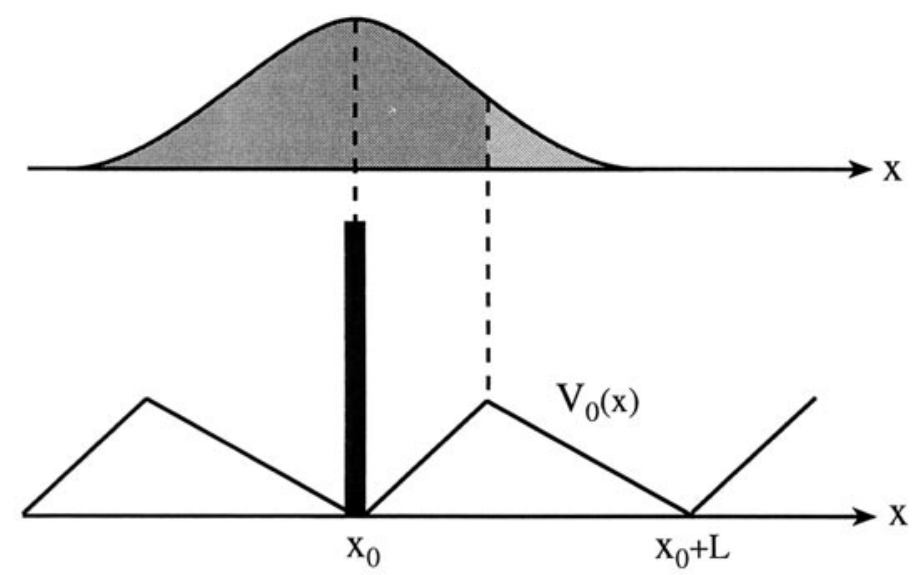

Fig. 2. Continuum analogue of the discrete games: the pulsating ratchet. The figure illustrates how Brownian particles, initially concentrated at $x_{0}$ in the presence of the potential $V_{0}(x)$, spread out symmetrically when this potential is switched off, but subsequently get captured mostly back in the basin of attraction of $x_{0}$, but also substantially in that of $x_{0}+L$. A net current of particles to the right, i.e., $\langle\dot{x}\rangle>0$, results.

Let us now start in the presence of the potential $V_{0}(x)$, and suppose that the Brownian particles are initially located at one of its minima, say at $x=x_{0}$, and that the thermal energy $k_{B} T$ is much smaller than the barrier height. Particles will thus in the presence of $V_{0}(x)$ mostly remain concentrated about the minimum $x_{0}$. After switching off the potential $V_{0}(x)$, the Brownian particles will start to diffuse symmetrically around $x_{0}$. When switching it on again at a later time, most particles will return to the minimum $x_{0}$, but a relatively large fraction will be attracted to the potential minimum at 
the right of $x_{0}$, cf. Fig.2. Hence a net flux to the right will develop. ${ }^{1}$ This systematic drift can now be used to perform work. ${ }^{2}$ At first, it may seem that the work which can be derived from the systematic drift of the particles is extracted from their Brownian motion. But one has to remember that the Brownian motor operates thanks to the switching-on and-off of the potential, and that by doing so, one creates nonequilibrium conditions in which there is a net work performed on the particles. We may view the switching-on and -off of the potential as a change of the thermal reservoir, with which the Brownian particles are in contact, between one at low temperature $\left(V_{0}(x)\right.$ on), and one at high temperature $\left(V_{0}(x)\right.$ off). In this presentation, the set-up resembles a Carnot engine. In fact, the efficiency of the pulsating ratchet can be calculated and is found to be much below Carnot efficiency. (Efficiency of ratchets have been studied by Vale and Oosawa 1990, Magnasco 1994, Jülicher and Prost 1995, Parrondo and Espanol 1996, Bier and Astumian 1996, Jülicher, Ajdari, and Prost 1997, Sekimoto 1997, Parrondo 1998a, Parrondo, Blanco, Cao, and Brito 1998b, Kamegawa, Hondou, and Takagi 1998.)

An important characteristic of the pulsating ratchet for the ensuing discussion is that it involves multiplicative noise: the potential that the particles feel can be written as $V(x, t)=V_{0}(x) \xi(t)$, where $\xi(t)$ is a stochastic process that switches at random between the values $1\left(V_{0}(x)\right.$ on) and $0\left(V_{0}(x)\right.$ off). Note that the amplitude of the noise, $V_{0}(x)$, depends on the location of the Brownian particle, hence the multiplicative nature of this noise. In the following two sections, we will present two surprising effects of multiplicative noise, which we will subsequently combine in a model of coupled Brownian motors in Sect.4.

\section{White Noise Ratchet}

A full analytic discussion of the pulsating ratchet subject to dichotomic and thermal noise for a general potential $V_{0}(x)$ is not available (special cases have been solved by Astumian and Bier 1994, Doering, Horsthemke, and Riordan 1994, Mielke 1995, Reimann and Elston 1996, Zapata, Luczka, Sols, and Hänggi 1998, Kula, Czernik, and Luczka 1998). We therefore turn to the case of a white noise driven pulsating ratchet $V(x, t)=\sqrt{2 Q} V_{0}(x) \xi^{\prime}(t)$, where $\xi^{\prime}(t)$ is a Gaussian ${ }^{3}$ white noise (in the sense of Stratonovich, see also footnote 5 below) and $Q$ characterizes the intensity of the potential

${ }^{1}$ A nice computer animation of this model is available on the internet under http://monet.physik.unibas.ch/ elmer/bm/

2 The resulting Brownian motor is sometimes referred to as "flashing-" or "onoff-ratchet". We think that the name "pulsating ratchet" is more appropriate, especially for the modified version of the model we consider below.

${ }^{3}$ White noise driven ratchets with sojourn times between kicks obeying a Poissonian statistics (white Poisson noise) have analytically been investigated by Luczka, Bartussek, and Hänggi 1995 and Luczka, Czernik, and Hänggi 1997. 
fluctuations (Reimann 1997). The thermal motion of the particles can be represented by an additive Gaussian white noise $\xi^{\prime \prime}$, independent of $\xi^{\prime}$, and with strength $\sqrt{2 T}$ (in units with $k_{B}=1$ ). The crucial advantage with respect to analytical tractability of this model is rooted in the well-known simple fact that the sum of two independent Gaussian random numbers is equivalent to a single one if one adds up the corresponding means and variances. This readily implies that the combination of independent Gaussian white noises $\xi^{\prime}$ and $\xi^{\prime \prime}$ can be replaced by a single Gaussian white noise term: ${ }^{4} a(x) \xi^{\prime}+b(x) \xi^{\prime \prime}=$ $\sqrt{a^{2}(x)+b^{2}(x)} \xi$. The motion of our Brownian particle is thus governed by the following stochastic differential equation (Reimann 1997): ${ }^{5}$

$$
\dot{x}=-V^{\prime}(x, t)=-V_{1}^{\prime}(x)+\sqrt{2 T+2 Q\left[V_{0}^{\prime}(x)\right]^{2}} \xi
$$

where $\xi$ is a Gaussian white noise characterized by its first two moments:

$$
\langle\xi(t)\rangle=0, \quad\left\langle\xi(t) \xi\left(t^{\prime}\right)\right\rangle=\delta\left(t-t^{\prime}\right) .
$$

Here we have included for generality also a non-fluctuating contribution $V_{1}(x)$ to the potential. $V_{0}(x)$ is the pulsating part of the potential with amplitude $Q$, and $T$ is the temperature experienced by the Brownian particle. The potentials $V_{0}(x)$ and $V_{1}(x)$ are both assumed periodic with period $L$. For earlier investigations of related problems see also Stratonovich 1969, Ivanichenko and Zil'berman 1969, Ambegaokar and Halperin 1969, Büttiker 1987, van Kampen 1988, Landauer 1988, Millonas 1995. A similar model in the context of Josephson junctions has been suggested by Berdichevsky and Gitterman 1997.

In view of the perfect periodicity of the system, it is sufficient to consider its behavior inside a "unit cell" $[-L / 2, L / 2]$ with periodic boundary conditions. One expects that the distribution of particles will attain a steady state in the long time limit with a steady but nonzero particle current. Both the steady state solution $P^{s t}(x)$ and the associated particle current $\langle\dot{x}\rangle$ are most easily obtained from the Fokker Planck equation (Risken 1984) associated to

${ }^{4}$ This is easily verified in the equivalent Fokker Planck equation description (cf. also footnote 5 and Risken 1984): $\frac{\partial}{\partial x} a \frac{\partial}{\partial x} a P+\frac{\partial}{\partial x} b \frac{\partial}{\partial x} b P=$ $\frac{\partial}{\partial x} \sqrt{a^{2}+b^{2}} \frac{\partial}{\partial x} \sqrt{a^{2}+b^{2}} P$. For $a(x)=\sqrt{D}$ and $b(x)=\sqrt{D^{\prime}}$ constant, this reduces to the well known result that the sum of two Brownian motions with respective diffusion coefficients $D$ and $D^{\prime}$ is again a Brownian motion with diffusion coefficient $D+D^{\prime}$.

${ }^{5} \mathrm{We}$ are considering here only the overdamped limit, i.e., inertia-effects are ignored, and the viscous friction coefficient has been absorbed into the time unit. Considering the white noise as limiting case of a colored noise with a correlation time that is negligible in comparison with all other relevant time scales of the system, it follows that the multiplicative white noise is to be understood in the sense of Stratonovich (Wong and Zakai 1965, Wong and Zakai 1969, Risken 1984). Furthermore, time-arguments have been dropped in this (and the later following) stochastic differential equation. 
the stochastic dynamics (1), with the following results (Stratonovich 1969, Ivanichenko and Zil'berman 1969, Ambegaokar and Halperin 1969, Büttiker 1987, van Kampen 1988, Landauer 1988, Millonas 1995, Reimann 1997):

$$
\begin{aligned}
P^{s t}(x) & =Z^{-1} \frac{\mathrm{e}^{-\phi(x)}}{g(x)} \int_{x}^{x+L} \mathrm{~d} y \frac{\mathrm{e}^{\phi(y)}}{g(y)} \\
g(x) & =\sqrt{T+Q\left[V_{0}^{\prime}(x)\right]^{2}} \\
\phi(x) & =\int_{0}^{x} \mathrm{~d} y V_{1}^{\prime}(y) / g^{2}(y) \\
\langle\dot{x}\rangle & =L Z^{-1}\left[1-\mathrm{e}^{\phi(L)}\right]
\end{aligned}
$$

where $Z$ is a normalization factor such that $\int_{-L / 2}^{L / 2} P^{s t}(x) \mathrm{d} x=1$. One concludes that not only the magnitude, but also the direction of the flux is a complicated function of the characteristics of the system. Note that the flux (6) is zero in the absence of multiplicative noise - in agreement with the fact that detailed balance is then reestablished - or in the case of symmetric potentials. In general, it is difficult to give an intuitive prediction of the flux direction. As an example, consider the case $V_{1}(x)=V_{0}(x)$. One easily sees from $(5,6)$ that the direction of $\langle\dot{x}\rangle$ is, for large temperatures $T$, dictated by the sign of $\int_{L / 2}^{L / 2}\left[V_{0}^{\prime}(y)\right]^{3} \mathrm{~d} y$ and for small temperatures by that of $-\int_{-L / 2}^{L / 2}\left[V_{0}^{\prime}(y)\right]^{-1} \mathrm{~d} y$. One expects that the two signs will, in many cases, be identical. But not necessarily so. In Fig.3, we schematically represent a very simple piecewise linear potential that can display a flux reversal upon variation of the temperature $T$ (Reimann 1997). Moreover, for $T$ large, it turns out (not shown in the plot) that, while the majority of particles is located to the left of the potential minimum, say at $x=x_{0}$, the flux is directed towards the right, in contrast to what happens for dichotomic noise, $\mathrm{cf}$. the discussion in the previous section. In other words, on average the particles surprisingly prefer to travel from their typical position $x<x_{0}$ down to the potential minimum at $x=x_{0}$ and then over the full barrier to their right rather than to directly surmount the partial remaining barrier that they typically see to their left.

In conclusion, the white Gaussian noise driven pulsating ratchet will, contrary to some previous suggestions (Magnasco 1993, Leibler 1994, Mielke 1995), typically display directed motion. The basic reason is that multiplicative white Gaussian noise is not equivalent to an (effective) thermal noise and generically breaks the symmetry of detailed balance. The flux direction is a complicated function of the parameters, including the possibility of flux reversals. For a more detailed discussion of this model we refer to Reimann 1997. 

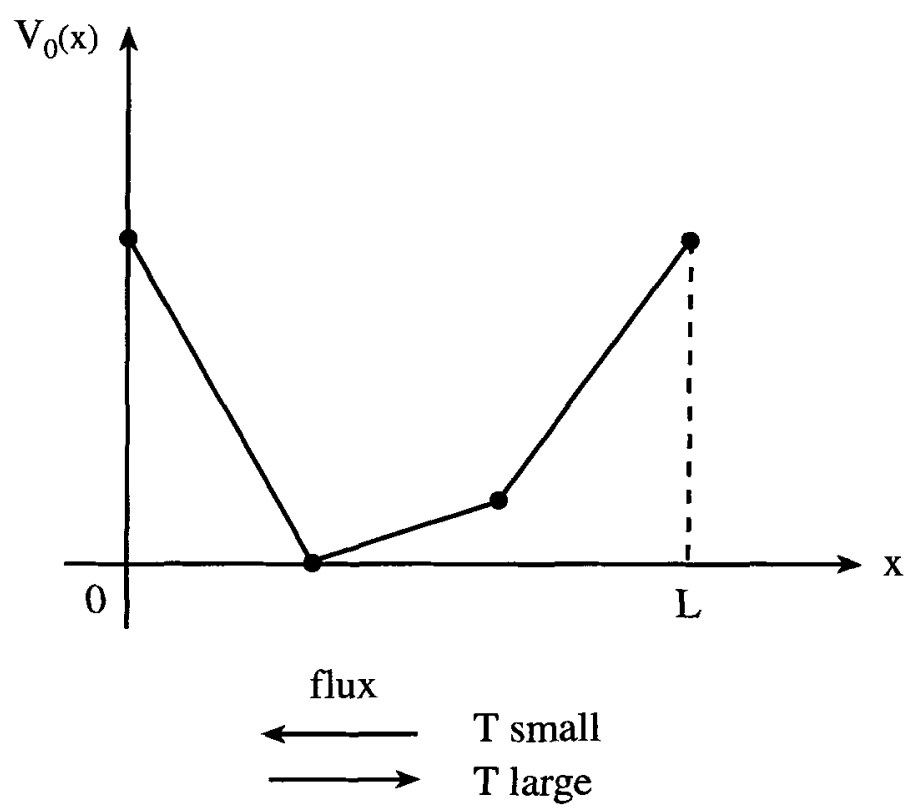

Fig. 3. Example of a piecewise linear periodic potential $V_{1}(x)=V_{0}(x)$ for which the corresponding white noise driven pulsating ratchet (1) experiences a flux reversal from $\langle\dot{x}\rangle<0$ for small $T$ to $\langle\dot{x}\rangle>0$ for large $T$.

\section{Noise Induced Phase Transitions}

The pulsating ratchet naturally involves multiplicative noise. Since it has been found recently (Van den Broeck, Parrondo, and Toral 1994, Van den Broeck, Parrondo, Toral and Kawai 1997, and further references therein) that such a multiplicative noise can induce genuine nonequilibrium phase transitions, displaying broken ergodicity and symmetry breaking, it is quite natural to investigate this possibility and its implications in the context of Brownian motors. To introduce the phenomenon of noise induced phase transitions, we turn to the simplest example of multiplicative noise, namely the Malthus equation for population growth with a fluctuating birth and death rate:

$$
\dot{x}=(-1+\sqrt{2 Q} \xi) x .
$$

Given an initial condition at time $t=0$, the solution reads: 


$$
x(t)=x(0) \exp \left\{-t+\sqrt{2 Q} \int_{0}^{t} \xi(\tau) \mathrm{d} \tau\right\} .
$$

Considering again the case of Gaussian white noise, $\mathrm{cf}$. (2), one easily verifies that $\int_{0}^{t} \xi(\tau) \mathrm{d} \tau$, which is nothing but the displacement of a free Brownian particle after a time $t$, is a Gaussian random variable with average zero and second moment equal to $t$. Hence this term is, with probability one, of the order of $\sqrt{t}$ and one concludes that $x(t)$ approaches 0 in the long time limit with probability one. On the other hand, one easily verifies from (8) and (2) that:

$$
\langle x(t)\rangle=x(0) \exp \{-t+Q t\}
$$

so that the average diverges for $Q>1$ ? The fact that typical and average behavior do not coincide is not really a surprise, just because of the multiplicative nature of the noise. Indeed, it is well known that the process $x(t)$ in (7) gives rise to a probability distribution with a long tail, and the behavior of $\langle x(t)\rangle$ is entirely dominated by the exponentially unlikely realizations in the tail. Hence, the obvious conclusion seems to be that this divergence is a mathematical peculiarity, and it will never (that is, with probability zero) be observed in, for example, a numerical simulation of (7). Furthermore, it turns out that the long-time limit of the first moment (9), and also of any higher moment, is regularized so as to approach zero by an arbitrarily small "saturation term", e.g., $\epsilon x^{3}$, on the right hand side of (7). In other words, nothing of interest then happens for any initial condition $x(0)$ and any strength $Q$ of the noise.

The situation is, however, more subtle when one considers a set of $N$ coupled elements $\left\{x_{i}, i=1, \ldots, N\right\}$, each obeying an equation similar to $(7)$ :

$$
\dot{x}_{i}=\left(-1+\sqrt{2 Q} \xi_{i}\right) x_{i}-\frac{K}{N} \sum_{j=1}^{N}\left(x_{i}-x_{j}\right)
$$

with independent Gaussian white noises $\xi_{i}$. For simplicity, we consider global coupling and take the thermodynamic limit $N \rightarrow \infty$. In this limit (Desai and Zwanzig 1978, Dawson 1983, Bonilla 1987, Strogatz and Mirollo 1991), each element $x_{i}=x$ is coupled to the mean field

$$
\langle x\rangle=\lim _{N \rightarrow \infty} \frac{1}{N} \sum_{j=1}^{N} x_{j}
$$

according to

$$
\dot{x}=(-1+\sqrt{2 Q} \xi) x-K(x-\langle x\rangle)
$$

from which $\langle x\rangle$ has to be determined self-consistently. The result of this simple analysis (Van den Broeck and Kawai 1998) is that, for any $K>0, Q=1$ is now the threshold for a nonequilibrium phase transition between a state 
of extinction $\langle x(t)\rangle \stackrel{t \rightarrow \infty}{\rightarrow} 0$ and explosion $\langle x(t)\rangle \stackrel{t \rightarrow \infty}{\rightarrow} \infty$. The interpretation is that the exceptional members of the ensemble, namely those that are responsible for the divergence of the first moment, become very influential through the global coupling and in fact drive the whole system into a new macroscopic state. In contrast to the single particle case, this explosion can now be easily seen in a computer simulation, even for relatively small sizes $N$. At first, one might still believe that the above situation is rather artificial, and maybe due to the peculiarities of the model under consideration. But it turns out that the appearance of such a noise-induced phase transition is a very robust phenomenon. It persists for nearest neighbor coupling in low dimensions (the present model in fact displays this phase transition even in $d=1$ ), in the presence of nonlinearities (e.g., a "saturation-term" $€ x_{i}^{3}$ ), and for other types of parametric perturbations including periodic ones (Van den Broeck and Kawai 1998). In fact, it can even appear for nonlinear models that have no instability in the absence of noise (Van den Broeck, Parrondo, and Toral 1994, Van den Broeck, Parrondo, Toral and Kawai 1997). Typically, the phase diagram becomes more complicated, including the appearance of a reentrance phenomenon with respect to the noise intensity and/or the strength of the spatial coupling.

The situation described in (10) is that of a pulsating harmonic potential. To transform this into a model for Brownian motors, we have to make a few modifications. First, we would like to have a periodic potential, but in contrast to "conventional" ratchet models, without any spatial asymmetry. This is easily achieved by replacing $x_{i}$ by $\sin x_{i}$ in the first term on the r.h.s. of (10). We will, mostly for the sake of analy tic tractability, also assume that the interaction has exactly the same periodicity and make the replacement $x_{i}-x_{j}$ by $\sin \left(x_{i}-x_{j}\right)$. Such a type of coupling appears for example in the planar $X-Y$ spin model (Arenas and Vicente 1994) or in models of phase synchronization of oscillators (Winfree 1980, Kuramoto 1984, Sompolinsky, Golomb, and Kleinfeld 1991, Swift, Strogatz, and Wiesenfeld 1992, Hansel, Mato, and Meunier 1993). Second, we need to include the thermal motion of the particles, which can be done by including an extra independent Gaussian white noise $\sqrt{2 T} \xi_{i}^{\prime}$ acting on each particle. As explained in the previous section, the white noises representing the pulsating ratchet and the one representing thermal motion can be combined into a single white noise contribution. We are thus led to the following model of coupled Brownian motors:

$$
\dot{x}_{i}=-\sin x_{i}+\sqrt{2 T+2 Q \sin ^{2} x_{i}} \xi_{i}-\frac{K}{N} \sum_{j=1}^{N} \sin \left(x_{i}-x_{j}\right) .
$$

One expects that the nonequilibrium phase transition out of the "absorbing" state ( $x=0$ modulo $2 \pi$ ) is still present in this system, since $\sin x=x$ for $x$ small. Furthermore, one expects that this transition is accompanied by a breaking of the $x \rightarrow-x$ symmetry, thus leading to an effective ratchet potential that produces a flux of particles (Jülicher and Prost 1995), notwithstand- 
ing the absence of a system-intrinsic preferential direction in (13). As it turns out (Reimann, Kawai, Van den Broeck, and Hänggi 1998), the symmetry breaking indeed takes place, however, without the appearance of a current, due to a hidden "accidental" symmetry in the model (13). We therefore turn, in the next section, to the discussion of a white noise driven pulsating ratchet in the presence of a somewhat more general pulsating potential than in (13).

\section{Collective Motion by Spontaneous Symmetry Breaking}

As a generalization of both (1) and (13) we consider the following model of coupled Brownian motors (Reimann, Kawai, Van den Broeck, and Hänggi 1998):

$$
\dot{x}_{i}=-V_{1}^{\prime}\left(x_{i}\right)+\sqrt{2 T+2 Q\left[V_{0}^{\prime}\left(x_{i}\right)\right]^{2}} \xi_{i}-\frac{K}{N} \sum_{j=1}^{N} \sin \left(x_{i}-x_{j}\right) .
$$

By exploiting $\sin \left(x_{i}-x_{j}\right)=\sin x_{i} \cos x_{j}-\cos x_{i} \sin x_{j}$ and with the definitions:

$$
\begin{aligned}
& \lim _{N \rightarrow \infty} \frac{1}{N} \sum_{j=1}^{N} \cos x_{j}=\langle\cos x\rangle=c \\
& \lim _{N \rightarrow \infty} \frac{1}{N} \sum_{j=1}^{N} \sin x_{j}=\langle\sin x\rangle=s
\end{aligned}
$$

one sees, similarly as in (10-12), that in the thermodynamic limit $N \rightarrow \infty$, every Brownian particle $x=x_{i}$ in (14) becomes coupled to a mean field (Desai and Zwanzig 1978, Dawson 1983, Bonilla 1987, Strogatz and Mirollo 1991) characterized by two order parameters $c$ and $s$ :

$$
\dot{x}=-V_{1}^{\prime}(x)+\sqrt{2 T+2 Q\left[V_{0}^{\prime}(x)\right]^{2}} \xi-K(c \sin x-s \cos x),
$$

where we dropped the subscript $i$ and where $c$ and $s$ have to be determined self-consistently from the solution $P(x)$ of $(16)$ with the definitions given in (15). Since (16) is a particular case of (1), the "steady state" properties can be copied from (3-6) with as only modification the replacement:

$$
V_{1}(x) \rightarrow V_{1}(x)-K(c \cos x+s \sin x) .
$$

This makes explicit our earlier statement that, for such a model with a perfect $x \rightarrow-x$ symmetry, a noise-induced symmetry breaking phase transition implying the appearance of a value $s \neq 0$ for this order parameter, will produce an effective asymmetric ratchet potential through the presence of the $s \sin x$ contribution in (17), making possible the appearance of a flux (Jülicher and Prost 1995). Again the flux can be copied from the general expression given 
in (6), and the only remaining difficulty is the solution of the self-consistent equations for $s$ and $c$, cf. (15) together with (3-6) and (17).

To illustrate the characteristic features of our coupled ratchet system (13), we concentrate on the following specific model:

$$
V_{0}(x)=V_{1}(x)=-\cos x-A \cos 2 x
$$

Note that for $A=0$, we recover the model (13). The additional term in $\cos 2 x$ does not break the $x \rightarrow-x$ invariance, but it removes the abovementioned "accidental" symmetry of the model (13). (Any other modification of the latter model will generically break this symmetry as well, for instance, potential fluctuations with a finite correlation in time.) As expected from the discussion in the previous section, the model (14) with (18) undergoes a noise-induced symmetry breaking phase transition with the appearance of $s \neq 0$ solutions. The symmetry breaking region was determined numerically from $(3,15,17)$ and is represented in Fig.4. The dependence of the associated flux (6) upon $A$ in this region of spontaneously broken symmetry is quite interesting: $A=0$ turns out to be the point of flux reversal. For $A<0$, the flux occurs in the same direction as the symmetry breaking (i.e., $s>0$ gives a flux to the right, while it is to the left if the symmetry happens to break to the other side, i.e. $s<0$ ). In contrast, for $A>0$, one has symmetry breaking and flux with opposite signs. ${ }^{6}{ }_{i}$ From the point of view of the mean field description (16), characterized by an asymmetric effective ratchet potential (17) for $s \neq 0$, this result is not really surprising since we mentioned before that the flux direction depends sensitively on the details of the model (see Fig. 3 and the discussion below (6)). But since the asymmetry here is produced by the effect of the symmetry breaking on the coupling term, which is a collective property, it has quite remarkable consequences (Reimann, Kawai, Van den Broeck, and Hänggi 1998).

First, we consider the response properties of the system, for $A>0$, with respect to a constant external force $F$. Such a force can be derived from an extra potential contribution $-F x$ to $V_{1}(x)$, and is tantamount to a tilting of the potential. A typical hysteresis curve is reproduced in Fig.5. It displays a remarkable anomalous behavior in comparison with the usual hysteresisloops, e.g., in a ferro-magnet, which can nevertheless be intuitively explained as follows. Consider the upper branch with a positive flux $\langle\dot{x}\rangle>0$. As expected, this flux increases upon acting with a force $F>0$. But at a critical

\footnotetext{
${ }^{6}$ A computer animation of this phenomenon is available on the internet under http://yukawa.phy.uab.edu/research/motor . It is based on simulations of $(14,18)$ with $N=1000, T=2, Q=4, K=10, A=0.15$ (cf. also Figs.4 and 5). Out of the 1000 particles, 100 are shown as green dots and one "tracer-particle" as a red dot. The position $x=-\pi$ is identified with $x=\pi$ (periodic boundary conditions). The initial particle distribution is symmetric about $x=0$. After a spontaneous breaking of the symmetry "to the right" $(s>0)$ an average particle current "to the left" $(\langle\dot{x}\rangle<0)$ can be observed.
} 


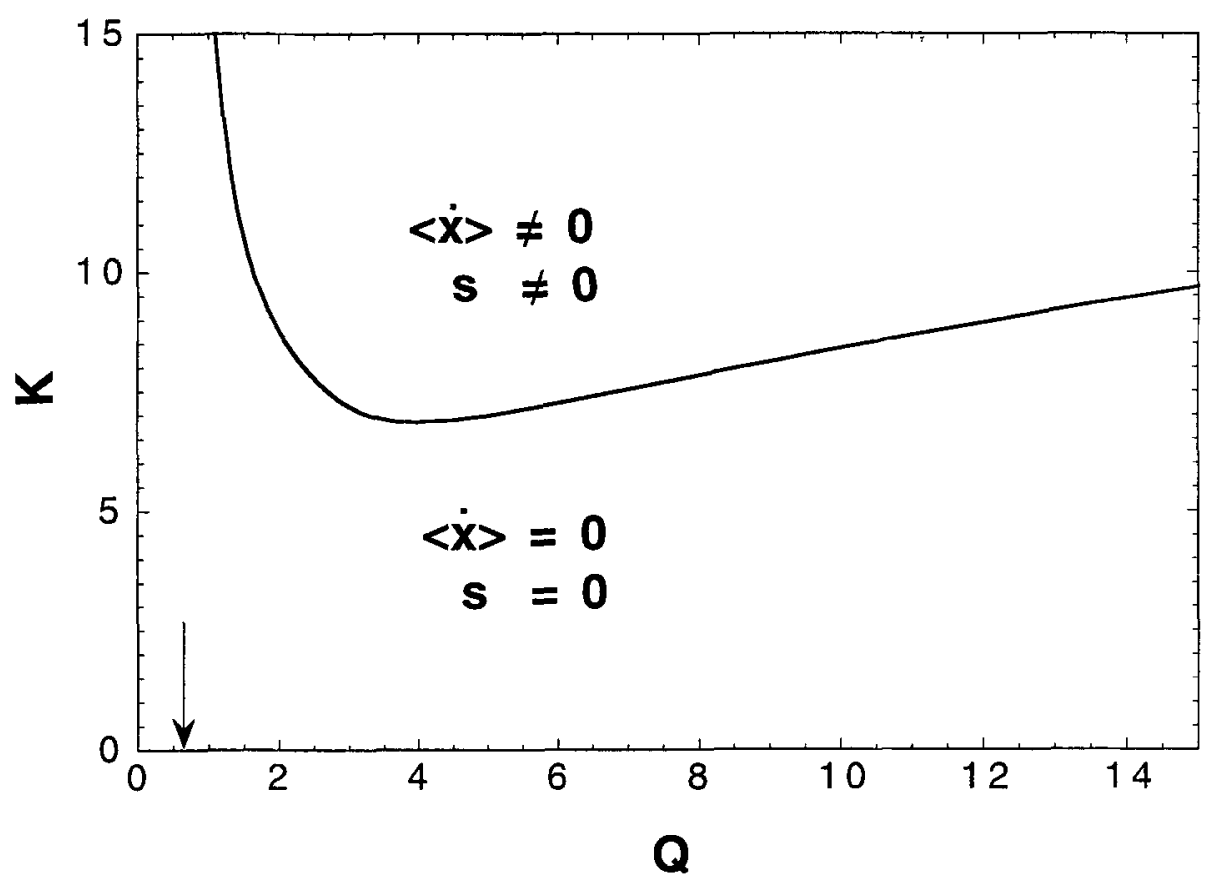

Fig. 4. Phase diagram for model $(14,18)$ with $T=2$ and $A=0.15$ in the thermodynamic limit $N \rightarrow \infty$, computed according to $(3-6,15,17) .\langle\dot{x}\rangle$ is the particle current and $s=\langle\sin x\rangle$ the order parameter (15) in the steady state. Arrow: asymptotic phase boundary for $K \rightarrow \infty$.

value of this force, the upper branch solution looses stability, and an abrupt transition to the lower branch, including a reversal of the flux to $\langle\dot{x}\rangle<0$, takes place. Put differently, given a spontaneous current in one or the other direction, we can apply a small additional force $F$ in the same direction with the expected result of an increased current in that direction. But upon further increasing $F$, the current will, all of a sudden, switch its direction and run opposite to the applied force! This astonishing feature clearly can only arise for an anomalous hysteresis-loop (Fig.5). To understand what is happening, we recall that $\langle\dot{x}\rangle>0$ for $F=0$ implies that one is in the symmetry broken state $s<0$, corresponding to a probability density $P^{s t}(x)$ that is concentrated in the region $x<0$ of the "fundamental cell" $[-L / 2, L / 2]$. However, as the potential is tilted to the right, this solution eventually looses its stability and the system switches abruptly to the only remaining steady state solution with $s>0$ and a strong flux, due to the specificities of the ratchet potential, to the left $\langle\dot{x}\rangle<0$. Note that upon a further increase of 
$F$, the stability of this solution is also eventually compromised (Fig.5) and a time-dependent state, characterized by oscillating flux, sets in through an infinite period bifurcations (Shinomoto and Kuramoto 1986).

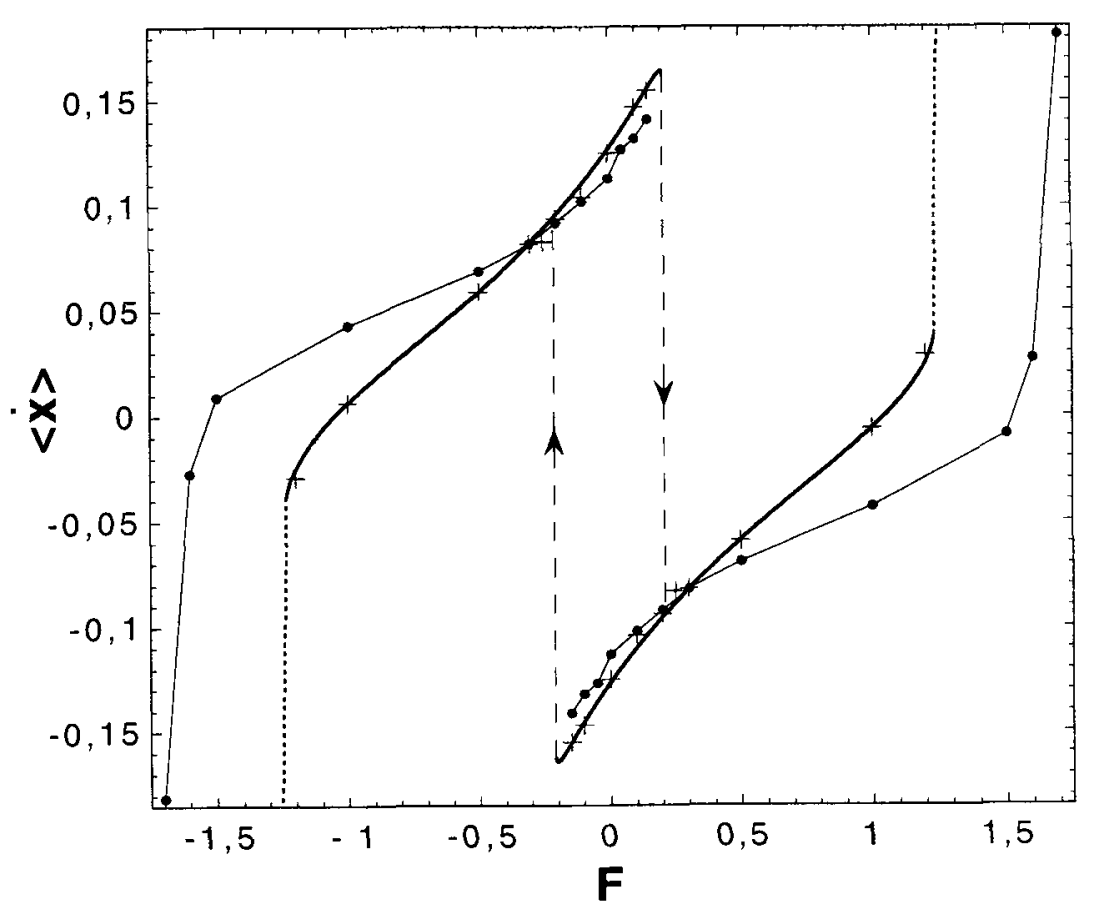

Fig. 5. Anomalous hysteresis-loops of the steady state current $\langle\dot{x}\rangle$ vs. force $F$ for model $(14,18)$ with $T=2, Q=4, K=10, A=0.15$. Solid: $N \rightarrow \infty$ by solving $(3-6,15,17)$. Crosses: simulations of $(14,18)$ for $N=1024$, averaged over 10 realizations. Interconnected dots: simulations for nearest-neighbor instead of global coupling in (14) on a $64^{*} 64$ square lattice with periodic boundary conditions and with modified parameters $Q=10, K=20$, averaged over 10 realizations. Dashed: time averaged current (for $N \rightarrow \infty$ ) in the regime of an oscillatory long time limit.

The mechanism explaining the anomalous hysteresis suggests another interesting question: can the symmetry breaking of a potential, induced by the mere action of an external force, produce a ratchet with a flux in the direction opposite to the force? In the case of a single particle, one easily concludes from (6) by including a term $-F x$ in $V_{1}(x)$, that the mobility $b=\left.\frac{\partial\langle\dot{x}\rangle}{\partial F}\right|_{F=0}$ is always positive, $b>0$, provided that the unperturbed situation displays no flux. Hence a force, applied to a zero flux situation, always induces, as 
expected, a flux in the direction of $F$. Considering however the case of coupled Brownian particles, it is clear that the effect of an external force is now two-fold. An extra term $-F x$ appears in the potential $V_{1}(x)$, but on top of that the interaction contribution $-K(c \cos x+s \sin x)$ in (17) also changes, since the presence of $F$ affects the steady state distribution $P^{s t}(x)$ of the particles. The following hand-waving argument turns out to be in agreement with a more detailed analysis (Reimann, Kawai, Van den Broeck, and Hänggi 1998). We consider $A>0$, and choose a set of parameters just outside the symmetry broken region. When applying a force, say $F<0$, the probability distribution $P^{s t}(x)$ will be deformed with a majority moving in the direction of the force, so that the average of the sin becomes negative: $s=s(F)<0$. As a result, each of the particles now perceives in addition to the symmetry breaking term $-F x$ a second symmetry breaking term $-K s(F) \sin x$. Furthermore, since one is close to the phase transition boundary with $s$ as the order parameter, one expects the usual divergence of the susceptibility, i.e., $s(F)=\chi F$ with $\chi$ positive and very large, suggesting that the deformation of the potential, $-K \chi \sin x F$, due to the indirect effect of the force on the distribution of the particles, is much larger than the the direct external force contribution $-x F$. The system is thus in essentially the same situation as when it is in the symmetry broken region $s<0$ in the absence of any force. But the ratchet mechanism then implies a flux in a direction opposite to $s$, hence opposite to $F$. In other words, we expect a negative zero-bias mobility which diverges as we get closer to the phase boundary (cf. Fig.4). This astonishing effect is indeed observed, cf. Fig.6. Furthermore, both the anomalous hysteresis and negative mobility persist when one replaces the mean field coupling by a 2-dimensional nearest neighbor coupling on a square lattice. Simulation results for these cases are included in Figs. 5 and 6.

\section{Discussion}

Though the ratchet effect, that is, the conversion of unbiased fluctuations into useful work, is a familiar every-day experience in many respects, in many others it is quite unexpected and intriguing. Our simple model from Sect.2 is especially appealing in that it allows a full analytical treatment, demonstrates that white Gaussian noise sources alone are sufficient, and reproduces the main effects known from more complicated models, as reviewed, e.g., in Hänggi and Bartussek 1996, Astumian 1997, Jülicher, Ajdari, and Prost 1997. ¿From those numerous studies it becomes increasingly clear that breaking thermal equilibrium, or equivalently, breaking the symmetry of detailed balance, in whatever way in a spatially periodic system, is generically sufficient for the ratchet effect to manifest itself. We thus more and more return to Feynman's point of view that the absence of directed transport in spite of a broken spatial symmetry is the truly astonishing situation. In general, the occurrence of a finite current should be the rule rather than the exception. 


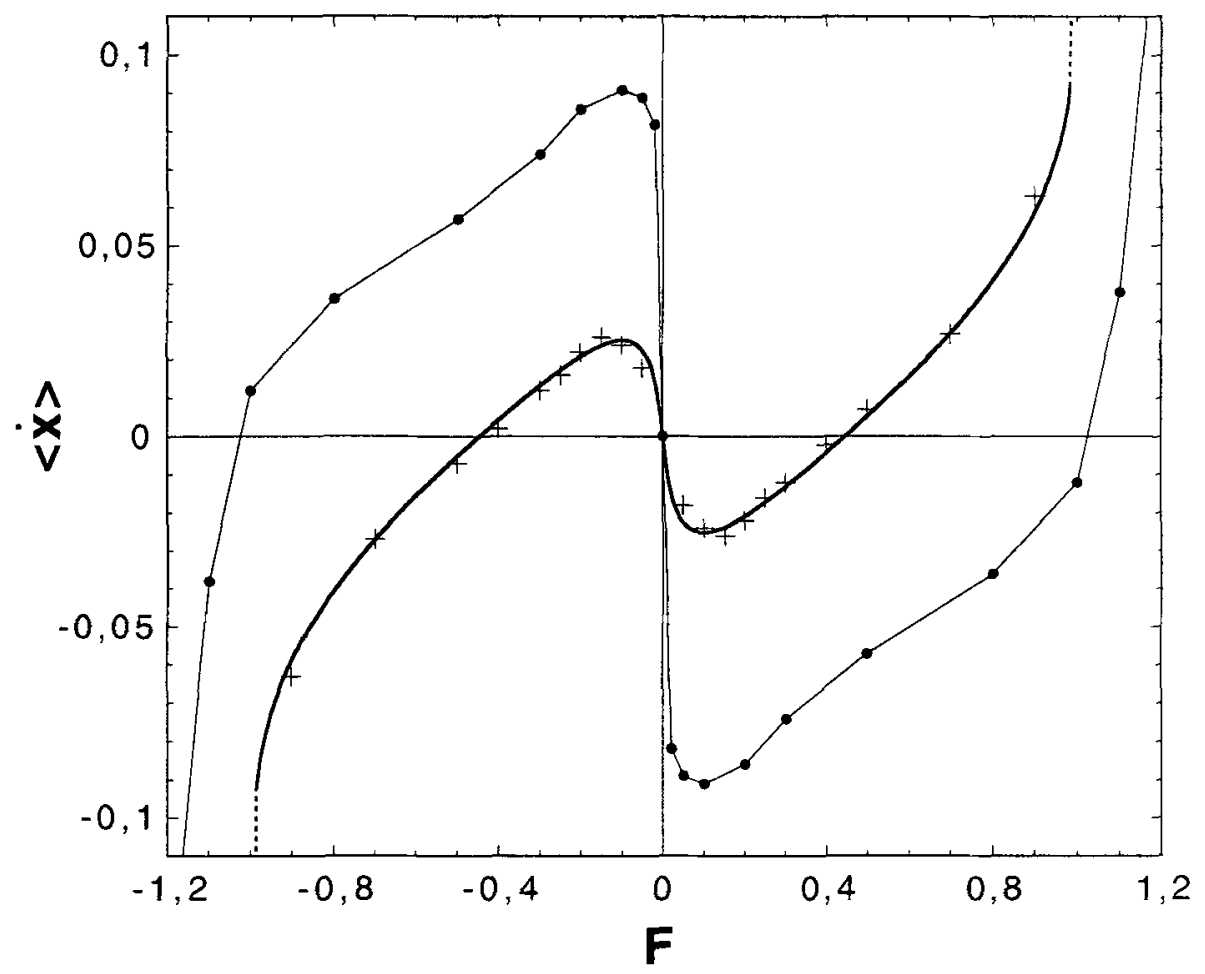

Fig. 6. Same as Fig.5 but for $Q=2, K=8$ (global coupling) and $Q=6, K=15$ (nearest neighbor coupling).

In Sect. 3 we reviewed a further "minimal model" for a completely different phenomenon induced by multiplicative noise when many Brownian particles are coupled, namely non-equilibrium phase transitions and breaking of ergodicity. Much like in a social context, a few very exceptional members may drag, by way of collective mechanisms, the whole system into a completely new state. Both, sufficiently strong (multiplicative) fluctuations and coupling are indispensable for such a phase transition.

Combining the ingredients of Sects.2 and 3 we finally came up in Sect.4 with a model for collective Brownian motors where, however, the usually built-in spatial asymmetry of the system is now substituted by a spontaneous symmetry breaking via a noise-induced non-equilibrium phase transition. Through the coupling, each particle then experiences an effective periodic potential with broken symmetry which, in concert with the non-equilibrium fluctuations, give rise to a directed spontaneous particle current in analogy to the "conventional" ratchet-effect. We remark that coupled Brownian motors have been discussed before in the literature by Derényi and Vicsek 
1995, Jülicher and Prost 1995, Marchesoni 1996, Häussler, Bartussek, and Hänggi 1997, Csahók, Family, and Vicsek 1997, Jülicher, Ajdari, and Prost 1997. Especially, Jülicher and Prost 1995 demonstrated in a somewhat different model of spatially symmetric, globally coupled Brownian motors the generation of a directed current by way of spontancous symmetry breaking and the closely related features of coexisting phases, first-order transitions, and normal hysteresis. The model that we introduced here, however, displays anomalous effects in response to an externally applied force that, to our knowledge, are completely new. Basically, they are the result of a competition between the effect of the external bias, favoring current in its direction, and the effective ratchet-potential that arises as a collective property and pumps particles in the opposite direction.

\section{Acknowledgments}

We would like to express our appreciation towards the organizers for the very stimulating and enjoyable XV Sitges Euroconference on "Statistical Mechanics of Biocomplexity", during which this work has been presented. We gratefully acknowledge support by the Program on Inter-University Attraction Poles of the Belgian Government, the F.W.O. Vlaanderen, the Deutsche Forschungsgemeinschaft (DFG-Sachbeihilfe HA1517/13-2), the Graduiertenkolleg GRK283 on "nonlinear problems in analysis, geometry, and physics", NATO grant CRG 950055, the Alabama Education and Research Network, and the Alabama Supercomputer Center.

\section{References}

Ajdari A. and Prost J. (1992): C. R. Acad. Sci. Paris 315, 1635

Ambegaokar V. and Halperin B.I (1969): Phys. Rev. Lett. 22, 1364

Arenas A. and Vicente C. (1994): Phys. Rev. E 50, 949

Astumian R.D. and Bier M. (1994): Phys. Rev. Lett. 72, 1766

Astumian R.D. (1997): Science 276, 917

Bartussek R., Hänggi P., and Kissner J.G. (1994): Europhys. Lett. 28, 459

Belinicher V.I. and Sturman B.I. (1980): Sov. Phys. Usp. 23(3), 199

Berdichevsky V. and Gitterman M. (1997): Phys. Rev. E 56, 6340

Bier M. and Astumian R.D. (1996): Bioelectrochemistry and Bioenergetics 39, 67

Bonilla L.L. (1987): J. Stat. Phys. 46, 659

Breymayer H.S., Risken H., Vollmer H.D, and Wonneberger W. (1982): Appl. Phys. B28, 335

Büttiker M. (1987): Z. Phys. B 68, 161

Csahók Z., Family F., and Vicsek T. (1997): Phys. Rev. E 55, 5179

Dawson D.A. (1983): J. Stat. Phys 31, 29

Derényi I. and Vicsek T. (1995): Phys. Rev. Lett. 75, 374

Desai R.C. and Zwanzig R. (1978): J. Stat. Phys. 19, 1

Doering C.R., Horsthemke W., and Riordan J. (1994): Phys. Rev. Lett. 72, 2984 
Feynman R.P, Leighton R.B., and Sands M. (1963): The Feynman Lectures on Physics, Vol. 1, Chapter 46 (Addison Wesley, Reading MA)

Hänggi P. and Bartussek R. (1996): in Lecture Notes in Physics, Springer-Series Vol. 476, Eds. Parisi J. et al. (Springer, Berlin)

Hansel D., Mato G., and Meunier C. (1993): Phys. Rev. E 48, 3470

Häussler R., Bartussek R., and Hänggi P. (1997): in Applied Nonlinear Dynamics and Stochastic Systems near the Millenium, Eds. Kadtke J.B. and Bulsara A., AIP Proc. 411

Ivanichenko Yu.M. and Zil'berman L.A. (1969): Zh. Eksp. 'Teor. Fiz. 55, 2395 [Sov. Phys. JETP 28, 1272]

Jülicher F. and Prost J. (1995): Phys. Rev. Lett. 75, 2618

Jülicher F., Ajdari A., and Prost J. (1997): Rev. Mod. Phys. 69, 1269

Kamegawa H., Hondou I., and Takagi F. (1998): Phys. Rev. Lett. 80, 5251

van Kampen N.G. (1988): IBM J. Res. Develop. 32, 107

Kula J., Czernik T., and Luczka J. (1998): Phys. Rev. Lett. 80, 1377

Kuramoto Y. (1984): Chemical Oscillations, Waves, and Turbulence (Springer, New York)

Landauer R. (1988): J. Stat. Phys. 53, 233

Leibler S. (1994): Nature 370, 412

Luczka J., Bartussek R., and Hänggi P. (1995): Europhys. Lett. 31, 431

Luczka J., Czernik T., and Hänggi P. (1997): Phys. Rev. E 56, 3968

Magnasco M.O. (1993): Phys. Rev. Lett. 71, 1477

Magnasco M.O. (1994): Phys. Rev. Lett. 72, 2656

Marchesoni F. (1996): Phys. Rev. Lett. 77, 2364

Mielke A. (1995): Ann. Phys. (Leipzig) 4, 476

Millonas M.M. (1995): Phys. Rev. Lett. 74, 10

Onsager L (1931): Phys. Rev. 37, 405

Parrondo J.M.R. and Espanol P. (1996): Am. J. Phys. 64, 1125

Parrondo J.M.R (1998): Phys. Rev. E 57, 7297

Parrondo J.M.R., Blanco J.M., Cao F.J., and Brito R. (1998): Europhys. Lett. 43, 248

Reimann P. and Elston T. (1996): Phys. Rev. Lett. 27, 5328

Reimann P. (1997): Phys. Rep. 290, 149

Reimann P., Kawai R., Van den Broeck C., and Hänggi P. (1998): preprint.

Risken H. (1984): The Fokker-Planck Equation (Springer, Berlin)

Schimansky-Geier L., Kschischo M., and Fricke T. (1997): Phys. Rev. Lett. 79, 3335

Seeger K. and Maurer W. (1978): Solid State Commun. 27, 603

Sekimoto K. (1997): J. of the Phys. Soc. of Japan 66, 1234

Shinomoto S. and Kuramoto Y. (1986): Prog. Theor. Phys. 75, 1105

Smoluchowski M.v. (1912): Phys. Zeitschr. 13, 1069

Sokolov M.I. and Blumen A. (1997): J. Phys. A 30, 3021

Sompolinsky H., Golomb D., and Kleinfeld D. (1991): Phys. Rev. A 43, 6990

Stratonovich R.L. (1969): Theory of Random Noise, Vol. 2, Chapter 9 (Gordon and Breach)

Strogatz S. and Mirollo R. (1991): J. Stat. Phys. 63, 613

Sturman B.I. and Fridkin V.M. (1992): The Photovoltaic and Photorefractive Effects in Noncentrosymmetric Materials (Gordon and Breach, Philadelphia) 
Swift J.W., Strogatz S., and Wiesenfeld K. (1992): Physica D 55, 239

Vale R.D. and Oosawa F. (1990): Adv. Biophys. 26, 97

Van den Broeck C., Parrondo J.M.R., and Toral R. (1994): Phys. Rev. Lett. 73, 3395

Van den Broeck C., Parrondo J.M.R., Toral R., and Kawai R. (1997): Phys. Rev. E 55, 4084

Van den Broeck C. and Kawai R. (1998): Phys. Rev. E 57, 3866

Winfree A.T. (1980): The Geometry of Biological Time (Springer, New York)

Wong E. and Zakai M. (1965): Ann. Math. Statistics 36, 1560

Wong E. and Zakai M. (1969): Wahrscheinlichkeitstheorie verw. Gebiete 12, 87

Wonneberger W. and Breymayr H.J. (1984): Z. Phys. B56, 241

Zapata I., Luczka J., Sols F., and Hänggi P. (1998): Phys. Rev. Lett. 80, 829 